\title{
Aberration Corrected STEM Imaging of Domain Walls in Congruent $\mathrm{LiNbO}_{3}$
}

\author{
Debangshu Mukherjee ${ }^{1}$, Greg Stone ${ }^{1, \mathrm{a}}$, Ke Wang ${ }^{2}$, Venkatraman Gopalan ${ }^{1}$ and Nasim Alem ${ }^{1}$ \\ 1. Department of Materials Science and Engineering, The Pennsylvania State University, University \\ Park, PA, USA \\ 2. Materials Characterization Laboratory, Materials Research Institute, The Pennsylvania State \\ University, University Park, PA, USA \\ a. Now at United States Army Armament Research, Development and Engineering Center, Picatinny \\ Arsenal, NJ, USA
}

$\mathrm{LiNbO}_{3}$ is a ferroelectric crystal at room temperature belonging to the $\mathrm{R} 3 \mathrm{c}$ space group, and has a Curie temperature of $1140^{\circ} \mathrm{C}$ [1]. The spontaneous electrical polarization of $\mathrm{LiNbO}_{3}$ is $71 \mu \mathrm{C} / \mathrm{cm}^{2}$ [2] while it's piezoelectric modulus, the $d_{33}$ value, is $31.5 \mathrm{pm} / \mathrm{V}$ [3]. The combination of high Curie temperature, electrical polarization and $d_{33}$ has led to applications as diverse as pyroelectric sensors, ferroelectric memory, quasi-phase matched second harmonic generators, optical switching etc. A multitude of these applications depend on the precise manipulation and control of domain walls in $\mathrm{LiNbO}_{3}$. The exact structure of the domain wall is however a subject of ongoing controversy on whether it is a pure Ising wall, or has a mixed Ising-Neel-Bloch nature [4]. In this work, for the first time we image domain walls with atomic resolution to measure displacements across them.

The origin of ferroelectricity in classical ferroelectrics like $\mathrm{LiNbO}_{3}$ is due to second order Jahn-Teller distortions where the B-site atom, which is niobium for $\mathrm{LiNbO}_{3}$, is displaced from the center of the oxygen cage [5], as shown in Fig la. This work focusses on probing the structural distortions and relaxation effects resulting from Jahn-Teller effect across the domain walls in $\mathrm{LiNbO}_{3}$ using aberrationcorrected scanning transmission electron microscopy, by imaging both oxygen and B-site atoms. A periodically poled congruent $\mathrm{LiNbO}_{3}(\mathrm{PPLN})$ crystal with $6.7 \mu \mathrm{m}$ wide y-wall $180^{\circ}$ domains were chosen for this study. The domain walls were imaged at a beam voltage of $200 \mathrm{kV}$ from the [010] pseudocubic orientation using annular dark field (ADF) and annular bright field (ABF) detectors simultaneously.

The ABF image (Fig. 1b) shows both the niobium and the oxygen atoms, with the niobium rows marked in yellow, and the oxygen positions marked in green in inset. The simultaneously obtained ADF image (Fig. 1c) shows just the niobium atoms from the same region. The domain wall becomes visible due to diffraction contrast in both the image sets. The niobium atom positions obtained from the ABF and the ADF images are cross-referenced with each other, while the oxygen atom positions are obtained from the ABF image. The combined atom positions are then used to measure the Jahn-Teller distortions and the spatial extent of the domain wall, which is compared with density functional theory simulations [6].

\section{References:}

[1] G. A. Srzolenski et. al. Phys. Stat. Sol. 13 (1966), p. 309

[2] A. Savage, J. Appl. Phys. 49 (1978), p. 3071

[3] M. L. Bortz, L. A. Eyres, and M. Fejer, Appl. Phys. Lett. 62 (1993), p. 2012

[4] D. Lee et. al. Phys. Rev. B. 80 (2009), p. 060102-1

[5] I.B. Bersuker, Ferroelectrics, 164 (1995), p. 75 
[6] This work was funded by the Penn State MRSEC, Center for Nanoscale Science, under the award NSF DMR-1420620.
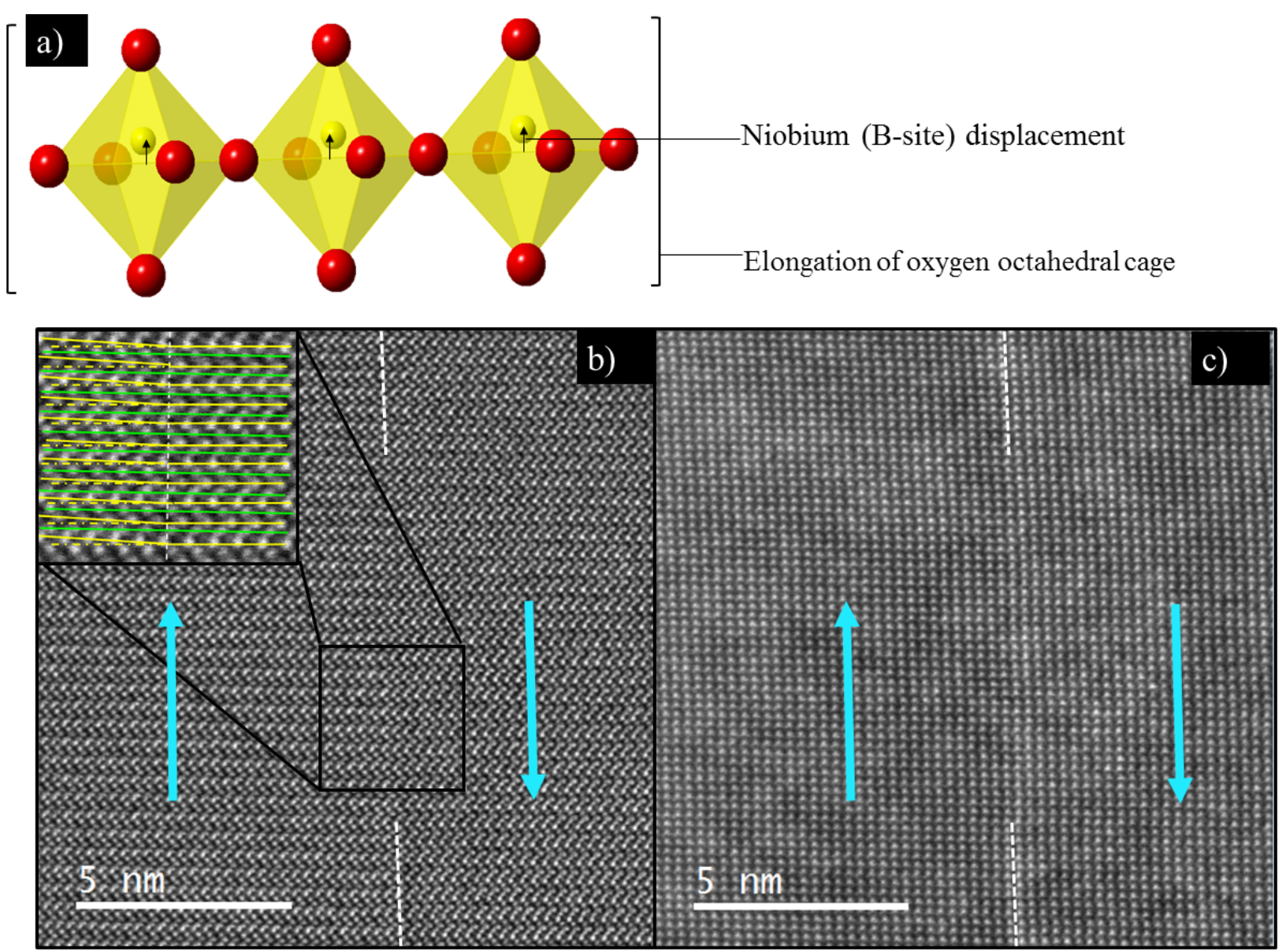

Figure 1. a) Schematic of second-order Jahn-Teller distortion, showing distortion of oxygen cages, with the arrows marking displacement of the niobium atoms from the center of the cage. b) ABF image of the domain wall, with the wall marked with white lines. The inset shows how the niobium rows marked in yellow bend across the wall (marked by the white dashed line), while the oxygen rows marked in green do not. The blue arrows refer to the polarization directions in the respective domains. c) ADF image showing the Niobium atoms across the domain wall. As in the ABF image, the blue arrows refer to the polarization directions in the domains. 